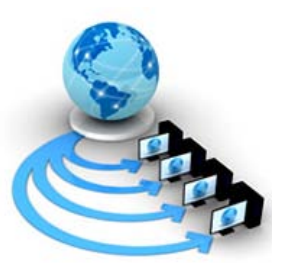

Volume 8, No. 8, September-October 2017

\title{
VM MIGRATION IN INNER CLOUD ON THE BASIS OF CLOUD LOAD USING MODIFIED ROUND-ROBIN
}

\author{
Ritika Songara \\ Research Scholar, \\ LNCTS(RIT)-Indore,India
}

\author{
Mayur Rathi \\ Assistant Professor, \\ LNCTS(RIT)-Indore, M.P, India.
}

\author{
Mayank Bhatt \\ HOD,Department of Computer Science \& Engineering \\ LNCTS(RIT)-Indore, M.P, India
}

\begin{abstract}
Cloud load balancing functionality divided into two sub functions. First function is resource allocation and second function is resources provisioning with task scheduling. Already many cloud load-balancing algorithms exist for load balancing. Algorithms are FCFS, Round-Robin, Equal load share and Throttled etc. Each algorithm has some disadvantage. We will implement VM migration on the basis of cloud load, into other inner cloud. We also calculate load of each inner cloud. In this paper, main focus on VM migration and implement cloud load balancing algorithm in inner cloud. We will implement VM migration and cloud load on java using CloudSim simulator.
\end{abstract}

Keywords: Cloud computing, Virtualization, Cloud load balancing.

\section{INTRODUCTION:}

Job Scheduling is a process of allocating jobs onto available resources in time. It is also defined as the process of finding an efficient mapping of tasks to the suitable resources so that the execution can be completed with the satisfaction of some objective functions. The objective functions could be such as minimization of execution time as specified by customers and maximization of resource utilization as specified by service providers. Efficiency of scheduling algorithm directly affects the performance of the system with respect to delivered Quality of Service. In short, more efficient is the scheduling algorithm, better is the Quality of Service delivered. Every Scheduling problem has three important elements [7]. They are:

Machine Configuration: A single machine with a single or multiple processors or a cluster of machines with a single or multiple processors in each machine etc.

Optimization Criterion: It defines the objective(s) of the scheduling algorithm e.g. reducing make span, minimizing response time, minimizing resource cost etc.

Set of constraints and characteristics: The scheduling of tasks may be dependent on some other tasks or independent of each other, thus defining a certain execution order and thus a certain set of constraints.

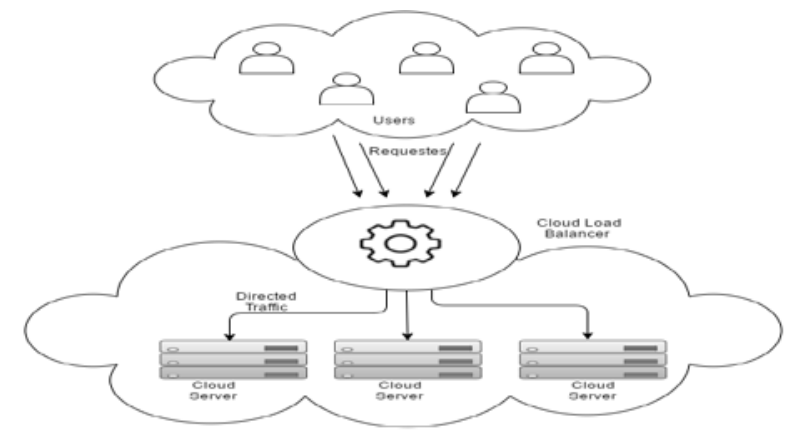

Figure 1: Cloud Load Balancer

Scheduling manages availability involving CPU memory and good scheduling policy gives maximum utilization of resource. Figure 1.4 shows that cloud load balancer.

\section{LITERATURE REVIEW}

Mohamed Riduan Abid et.al.[1] in this paper they have given the drivers behind the demanding would like for a load-balancer in Inter-Clouds environments, and delineated its robust correlation with virtualization. we have a tendency to plan a unique VM migration scheme impressed from the mobile computing handoff mechanism. Besides, we have a tendency to give a blueprint, then will be easily adopted in the domain, for deploying a real-world Inter-Cloud testbed using ASCII text file code. The Inter-Cloud testbed will be used to further investigate Inter-Cloud Load-balancing relevant analysis problems, e.g., VMs live Migration.

In paper [5] a novel VM fill up balancing rule is really Weighted Active observation populate reconciliation rule applying CloudSim tools, because of the Datacenter to 
assist with efficiency load balance requests between ones exhibited virtual devices distribution the weight, so as to come through much better performance parameters. Here VMs associated with completely different process powers together with the tasks/requests typically are selected or may be issued on all-powerful VM on the lowest so on.

In paper [6] author projected a good rule will be ant colony optimization that random optimization search approach is typically obtained pertaining to allocating your current incoming jobs on the virtual machine.

In the Current scenario, with surroundings of mobile cloud, the task is divided and disseminated into the same size of little jobs i.e. Cloudlets. These Cloudlets, as well as Virtual Machines, are scheduled according to the varied programming policy for e.g. FCFS, RoundRobin etc. usually in Cloud Computing situation user submit the task to be performed / dead. Cloud organizer (CC) [2] divides the task into equal sized cloudlets and passes it to DataCenter (DC). Normally it takes a lot of time as a result of the cloudlets is processed one at a time in FCFS manner as and once they reach to VM. VM executes the cloudlets present in the queue as they reach the VM's. Essentially this default job scheduled policy is extremely Time- consuming, value insensitive and inefficient.

\section{PROBLEM IDENTIFICATION:}

"Cloud computing is a term, which involves virtualization, distributed computing, networking, software and web services”. As we talk about a cloud it consists many parameters like shoppers, datacenter \& distributed system. Cloud comprises of fault tolerance, convenience, and quantifiability, litheness, compact overhead for users, compact value of possession etc. [2].

The core to this crisis lays institution of efficient load equilibrium formula. The weight may be processor, space capability etc. [4]. Load balancing is therefore may be defined as the method of allocate the load among different nodes of a DS to enhance each resource employment and process latency whereas additionally avoiding a state of affairs wherever a number of nodes are highly loaded whereas alternative nodes are idle or doing little or no work, and some of the physical machines and virtual machines are having maximum imbalance level of Cloud data centers [5].

\section{SOLUTION DOMAIN}

Our aim is implement Round Robin and Modified RoundRobin with Priority scheduling policy for VM using Cloudsim3.0. We will also implement combination of load balancing algorithms like Round Robin with priority and less resources first.

\section{Proposed Algorithm:}

1. Creates same size of Cloudlets.

2. CC divides the assigned Cloud task into same size of cloudlets.

3. Create Broker and User assigns the task to Cloud Coordinator (CC).
4. CC sends cloudlets to VMM and VMM sends the list of the needed resources to the RsP.

5. Request for the execution of the Cloudlet is sent to the VM by VMM from the Host.

6. Set all the VM allocation is zero. After set Parameters

7. Calculate Range using below formula Range=Time (Max Burst Time) + Time (Min Burst Time).

8. On the basis of Range set Priority of Cloudlets.

9. After the complete of task (cloudlets), VM are allocated to other user request.

10. Cloudlet scheduling is done in VM according to shortest job first then round-robin scheduling policy.

11. Sends the executed job as Cloudlets in a wrap file to the VMM.

12. VMM further passes the executed Cloudlets as wrapped file format to CC.

13. CC combines all executed Cloudlets in wrapped file form combine to form the whole task.

14. CC sends the executed task in authenticated file format to the user/client.

15. PRINT the Result.

\section{RESULTSET}

Cloud load balancing is developed in this research with help Java (JDK1.8) and Net Beans IDE8.02 on window operating sytem7. All forms of Cloud load balancing System design in Swing. Graph plotted for computation time and memory management using JFree Chart Library. In Result Analysis compare Proposed system with existing system in term of computation time and memory.

First step: This is the first page of our project, which is shown in figure1.

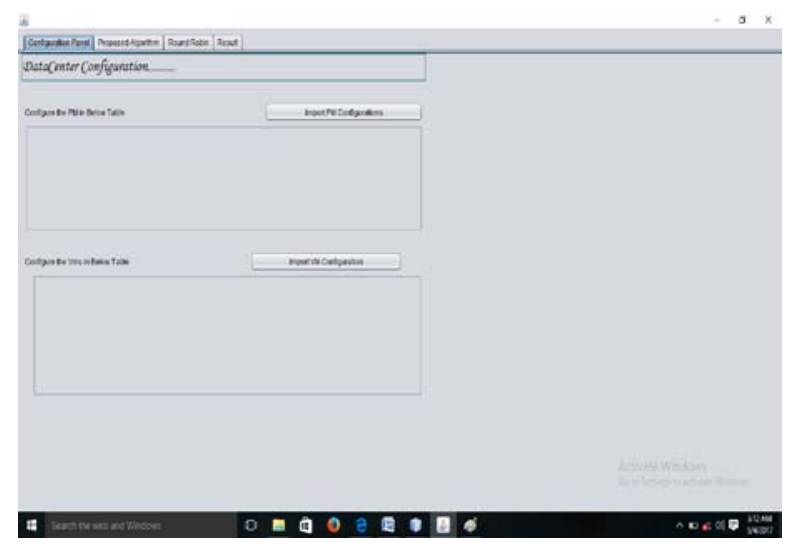

Figure 1: Demonstration of proposed work First Step

Second Step : After doing first step user import the PM, user import the PM from the available allocation of the PM which is shown in figure2. 


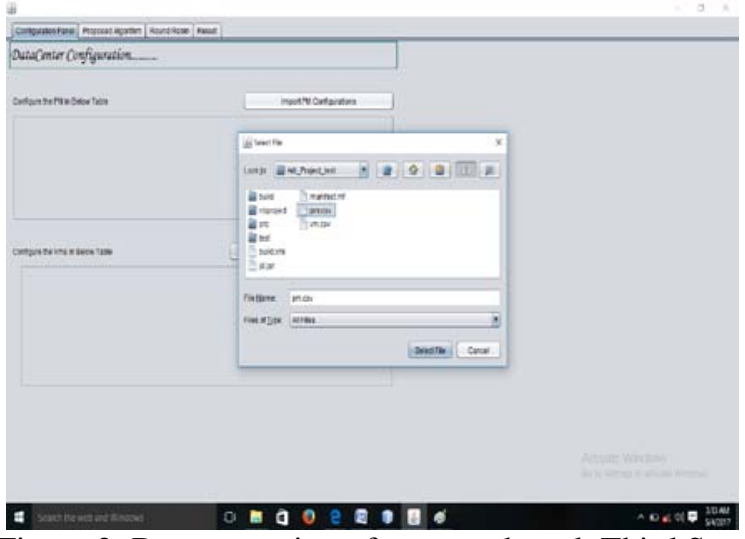

Figure 2: Demonstration of proposed work Third Step

Third Step: After import the PM Now imports the VM which is created from the imported PM here VM are show in figure 3.

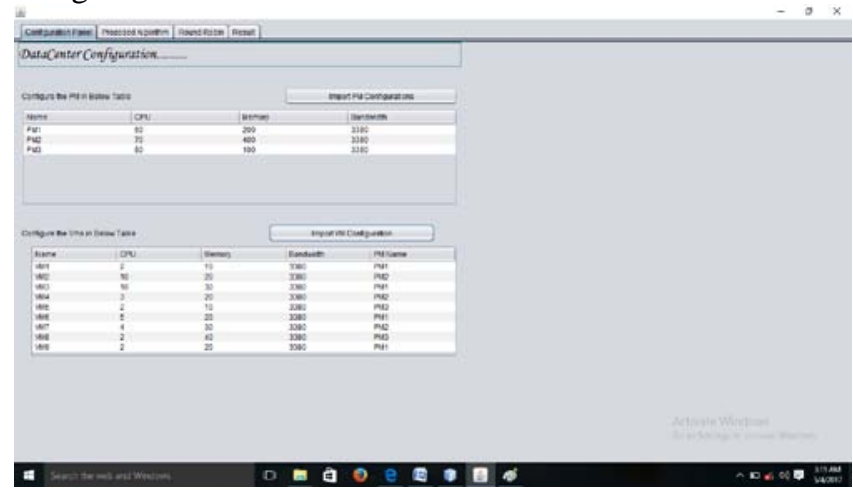

Figure 3: Demonstration of proposed work Third Step

Fourth Step: Now we are compare both the proposed and round robin algorithm we are click on the proposed algorithm button and then click on the process button for result which is show in fig 4 .

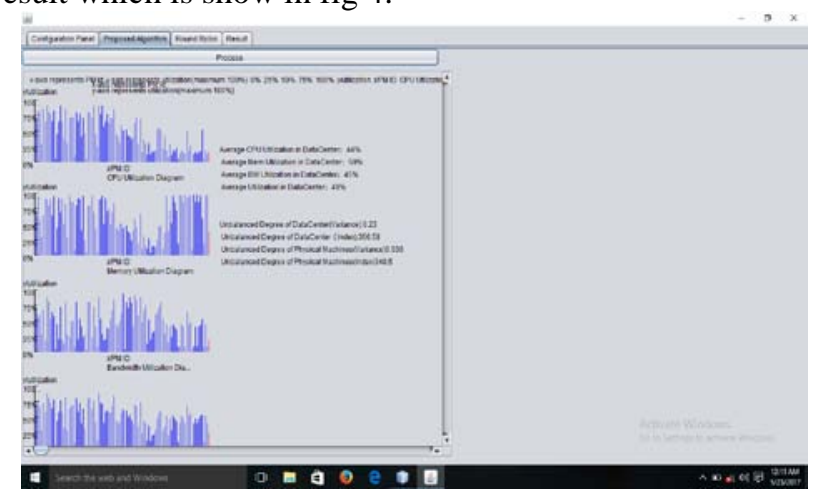

Figure 4 : Demonstration of proposed work Fourth Step

Fifth Step: Same for looking the result of the round robin we click on the round robin button for result, which is show in the fig 5

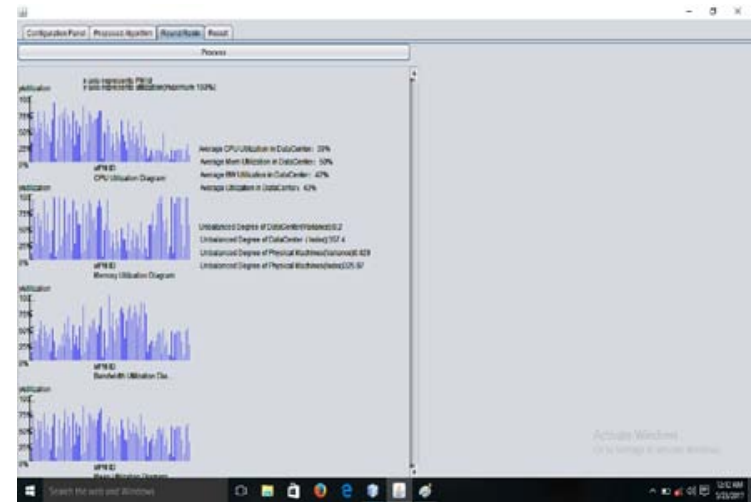

Figure 5: Demonstration of proposed work Fifth Step

Sixth Step: At the same time for looking the comparing result we are click on the result button which is show on fig 6.

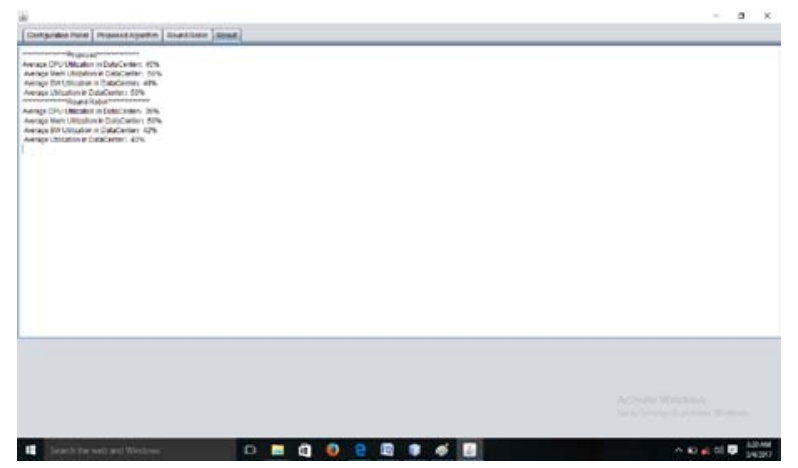

Figure 6 : Demonstration of proposed work Sixth Step

\section{CONCLUSIONS AND FUTURE WORK:}

In this paperwork on cloud load balancing algorithm. Proposed new algorithm for load balancing. The major thrust is given on the study of load balancing algorithmic rule, followed by a comparative survey of these on top of mentioned algorithms in cloud computing with reference to stability, resource utilization, static or dynamicity, cooperative or non-cooperativeness and method migration. This paper aims towards the establishment of performance analysis on existing VM load balancing algorithmic rule and so implemented in CloudSim and java language. In Future either improved algorithm or combination of algorithms will improve performance of cloud load balancing. In Future also compare load-balancing algorithms on other parameters.

\section{REFERENCES:}

[1] Mohamed Riduan Abid, Moulay Idriss El Ouadghiri, Michael Gerndt “Virtual Machines' Load-Balancing in Inter-Clouds” 2016 4th International Conference on Future Internet of Things and Cloud Workshops , (C) 2016 IEEE DOI 10.1109/W-FiCloud.2016.35

[2] Rajkumar somani, Jyotsana Ojha, “A Hybrid approach for Vm load balancing in cloud using cloudsim”,2014,International Journal of science, Engineering and Technology Research(IJSETR),Volume 3.Issue6,June 2014

[3] .Ritu Kapur, "A Cost Effective approach for Resource Scheduling in Cloud Computing”, 2015, IEEE International Conference on Computer,Communication and Control(IC42015). 
[4]. Dr. Rakesh Rathi1, Vaishali Sharma2 and Sumit Kumar Bole3, "Round Robin Data Center Selection in Single Region for Service Proximity Service Broker in Cloud Analyst” , International Journal of Computer \& Technology, Volume 4 no. 2, March- April 2013.

[5]. Bhatiya Wickremansinghe1, Rodrigo N. Calheiros2and Dr. Rajkumar Buyya3, "CloudAnalyst: A CloudSim- based VisulModeller for Analysing Cloud Computing Environments and Applications”, IEEE Computer Society, 2010.
[6]. Kunal Mahurkar1, Shraddha Katore2 and Suraj Bhaisade3, Pratikawale4, "Reducing Cost of Provisioning in Cloud Computing”, International Journal of Advance in Computer Science and Cloud Computing, Volume- 1, Issue- 2, nov.2013,

[7] . Syed Tauhid Zuheri1, Tamanna Shamrin2 and Rusia Tanbin3, Firoj Mahmud4, “An Efficient Load Balancing Approach in Cloud Environment by using Round Robin Algorithm”, International Journal of Artificial and Mechatronics, volume 1, issue 5, 2013. 